Working Papers on The Nature of Evidence:

How Well Do 'Facts' Travel?

No. $38 / 10$

\title{
Designed for Travel: Communicating Facts Through Images
}

Martina Merz

(C) Martina Merz

University of Lucerne 


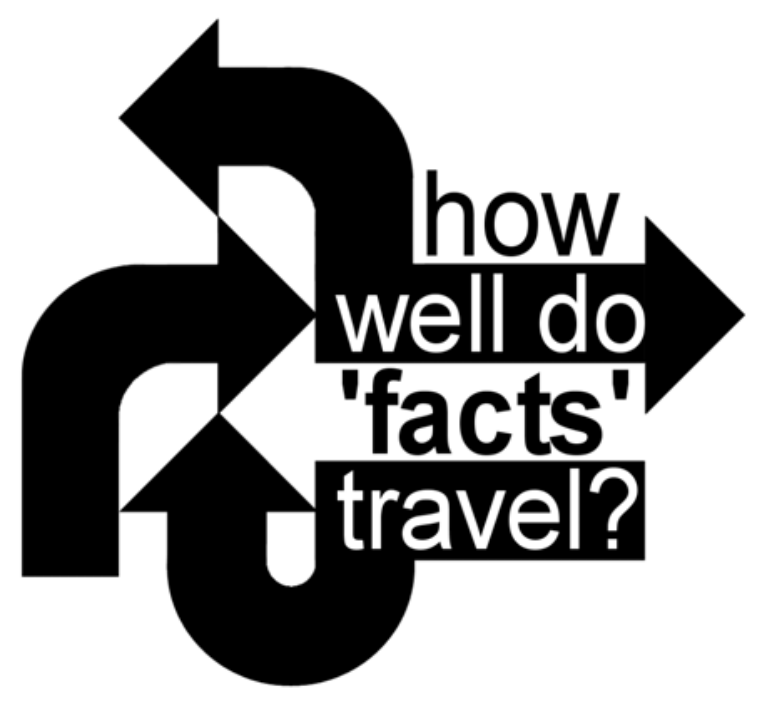

"The Nature of Evidence: How Well Do 'Facts' Travel?" is funded by The Leverhulme Trust and the ESRC at the Department of Economic History, London School of Economics.

For further details about this project and additional copies of this, and other papers in the series, go to:

http://www.Ise.ac.uk/collection/economichistory/

Series Editor:

Dr. Jonathan Adams

Department of Economic History

London School of Economics

Houghton Street

London, WC2A $2 \mathrm{AE}$

Tel: $\quad$ +44 (0) 2079556727

Fax: $\quad$ +44 (0) 2079557730 


\section{Designed For Travel: Communicating Facts Through Images ${ }^{\dagger}$}

Martina Merz

\section{Introduction}

Visual images can be effective devices for communicating facts. ${ }^{1}$

Yet this does not imply that whenever images propagate the facts automatically come along - nor do facts that travel in images always travel well. The relation of images, facts and their travels is more complex. The complex relationship will be explored in this text for the case of microscopy images in the field of nanotechnology and their travels both through scientific publications and popular media.

Nanotechnology researchers produce images by using probe microscopy, such as scanning tunnelling microscopy (STM) and atomic force microscopy (AFM), and electron microscopy. ${ }^{2}$ Unlike optical microscopy, which resolves structures in the range of millimetres and fractions thereof, these types of microscopy operate at the level of atoms and attain atomic resolution. Scientists use the instruments to image and analyze atomic and molecular structures. But importantly, probe

\footnotetext{
+ The research underlying this paper was funded by the Swiss National Science Foundation as part of the project "Epistemic Practice, Social Organization, and Scientific Culture: Configurations of Nanoscale Research in Switzerland". It has also benefited from generous funding provided by the Leverhulme Trust/ESRC grant "The Nature of Evidence: How Well Do 'Facts' Travel?" in the context of two extended research stays with the "Travelling Facts" team at the LSE in London. I thank the Facts team, the participants of the book workshop at LSE, and, especially, the book's two editors for their constructive criticism. I am grateful to the nanotechnology researchers for introducing me into the intricacies and routine tasks of their research.

${ }^{1}$ In accordance with constructivist science studies this article takes as a fact what is established as a fact through material and discursive practice within an epistemic community.

${ }^{2}$ A note on terminology: the notion "image" refers to visual images only and not to other kinds of images such as metaphors. "Nanotechnology" is employed in this text as a synonym for both nanotechnology and nanoscience. This choice is motivated, first, by a preference to increase readability and, second, based on the understanding that the distinction between the two is often used in contingent ways in the concerned communities.
} 
microscopes also allow researchers to produce and manipulate such nanoscale structures. Through the exploitation of quantum mechanical effects, these instruments are employed to produce objects (e.g. materials) with novel properties. This potential and practice is considered a defining and characteristic constituent of nanotechnology (cf. Baird et al. 2004; Daston and Galison 2007, chap. 7; Hennig 2009; Mody 2004).

The lab-produced images of atomic or molecular structures are among the most important outcome of nanotechnology practice. A small selection of these lab images, suitably edited, has found their way into scientific publications through which the researchers communicate their findings to their peers. An even smaller selection of the images, edited in other ways, has been diffused through alternative channels (news media, websites, etc.) to the public. ${ }^{3}$ Images that originate in scientific laboratories carry facts. Scientists package facts of different kinds in the form of images and visual displays to transfer them from their context of production - the scientific laboratory - to other contexts. ${ }^{4}$ How these packages are designed for travel and how users unpack them later on is in the focus of this article.

Within and across the scientific field images do not travel easily on their own. To travel well, they require good company: labels and instructions for use, an accompanying explanatory or contextual text. ${ }^{5}$ But above all, they are rarely to be found without the companionship of related images or other visual representations. The travelling companions are not just there for the ride, but are essential epistemic elements in the

\footnotetext{
${ }^{3}$ Besides images that originate in the scientific laboratory, a wide range of other images has become associated with nanotechnology in popular media (Landau et al. 2009; Lösch 2006; Milburn 2008; Nerlich 2008); such images will not feature in this article.

${ }^{4}$ The case of Calhoun's images associated with his rat experiments, discussed by Ramsden in this volume, offers an example of this.

${ }^{5}$ See Leonelli (this volume Simona: please supply full ref) for a related discussion on the issue of labels and packaging.
} 
way that the scientific culture of nanotechnology produces and communicates facts. In contrast, the diffusion of images from science into other spheres follows its own rules and guidelines. Images may be stripped of their companions, become iconised or recontextualised in novel ways. Thus, whether facts travel well by unaccompanied images is judged according to distinct standards of evaluation in different communities.

In the following, the case of an emblematic image from nanotechnology that has been diffused widely within the public realm will feature first (section 2). I will next turn to microscopy images in research articles, to their packaging in composite visual displays and to the role images play in the transferral of facts from an article's authors to its scientific readers more generally (section 3 ). How a composite visual display is unpacked by a fellow scientist is analyzed to illustrate the factimage travel dynamics (section 4). The text concludes with a discussion of the sense in which facts travel well by way of images (section 5).

\section{The IBM Logo: Facts, Images, Icon}

One of the images most closely associated with nanotechnology in the public imagination is the IBM logo. It can be downloaded from a variety of internet sites as shown in fig. 1 - i.e. with specific contrasts, shapes and colours - and it frequently appears in print media (Baird and Shew 2004; Hennig 2009). This image will be introduced first from the perspective of its viewers before turning to the question of the image's scientific origins.

\subsection{Dissemination in the Public Realm}

What is the image about? To assess the response of viewers to this question, I confronted twenty people of different professional and 
educational background with a colour print of the image that contained neither a legend nor any other additional information. It turned out that a majority of the people had not previously seen the image. These first-time viewers identified the image merely with the word "IBM", the company's

\section{Figure 1. IBM Logo Composed of Individual Atoms}

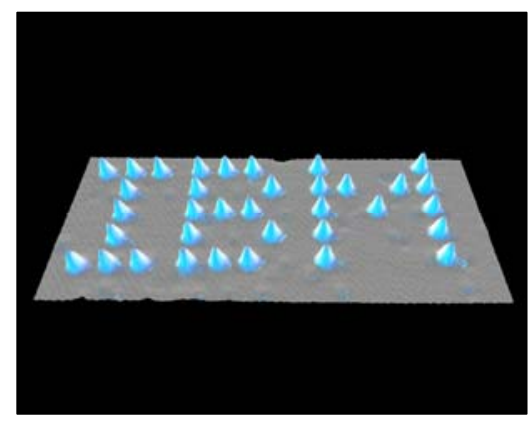

Image originally produced by IBM

logo. This answer came in variations, e.g. "the brand IBM", "IBM: the computer producer (logo)" or "publicity for IBM". Other respondents associated the image with the process of its production, e.g. "writing produced by 'nano'-technology", "representation by a nano-microscope", and "a small joke from the IBM research lab". This modest assessment suggests that the viewers' reading of the IBM image decisively depends on whether they have been previously exposed to it and on the availability of background information. Assuming that viewers have such background knowledge, what is the image about? This text argues that an important reason why the IBM image has come to symbolize nanotechnology is that crucial facts about the power of nanotechnology are associated with the image - but for the facts to actually reach the viewer (that is, for the facts to travel well, see below), the image needs to be accompanied by additional information. This information may come in the form of a legend or of accompanying text in another format. Consider an example from the 
website of the British Science Museum ${ }^{6}$. The Museum's website guides online visitors through the antenna "Nanotechnology: small science, big deal" to the rubric "See for yourself", from there to the "Exhibition sneak preview", where the IBM logo (as shown in fig.1) is exhibited under the header "The smallest world" with the legend "Each blue blob is a xenon atom arranged using a microscope." In addition, the image is accompanied by the text:

Each blue blob in this image is a xenon atom. Scientists working for IBM used a scanning tunneling microscope to move the atoms around and write their company logo. Each atom is one tenth of a nanometre wide, so this entire word could be written 14 million times onto a stamp. ${ }^{7}$

A closer look at this material leads to the proposition that more than one kind of fact is involved. The combination of image, legend and supplementary text conveys three types of factual statements. The building blocks of the letters I, B and $\mathrm{M}$ consist of individual atoms, in this case xenon atoms, the scale of the entire composition being in the range of nanometres - this constitutes a fact about the imaged phenomenon (phenomenal fact). The atoms were moved into place by a scanning tunnelling microscope (STM) - this represents a fact about the employed course of action to produce the phenomenon (procedural fact). An STM has been used both to move atoms and to visualize the result of this manipulation - this is a fact about the apparatus and technology used (technological fact). ${ }^{8}$

Once the reader is aware that the blobs represent single atoms, the image unfolds its suggestive power: because the pattern $(I, B, M)$ is so

\footnotetext{
${ }_{7}^{6}$ www.sciencemuseum.org.uk

${ }^{7}$ www.sciencemuseum.org.uk/antenna/nano/seeforyourself/153.asp (seen May 2, 2009).

${ }^{8}$ Howlett and Velkar (this volume Simona, please supply full ref) also use the term 'technological fact' in this way.
} 
manifestly artificial, impossible to imagine as a product of anything but an intentional act, the procedural fact is inscribed in it as much as the fact that the technological capacity exists to perform the task. The accompanying text only adds the details: that an STM was used, that xenon atoms were placed on a nickel surface. The replies of the second set of respondents (above) point at this association of the IBM logo with procedural and technological facts.

Calling the image "a small joke from the IBM research lab", as another respondent above did, identifies it with the corporation's scientific and technological project. But more than merely the trace of a joke, the image is a forceful reminder of and renders homage to the company behind the project. The instrument used to produce the sample, the STM, had been invented by IBM researchers $\mathrm{G}$. Binnig and $\mathrm{H}$. Rohrer, who received the Nobel prize for this invention in 1986, whilst other researchers of the corporation, D.M. Eigler and E.K. Schweizer, had produced the nano-scale IBM logo in their lab (Binnig et al. 1982; Hennig 2004, 2006).

To reiterate, while the IBM logo image is widely diffused, it requires an accompanying text to ensure that the procedural and technological facts travel with it and are well received. In the terminology of this volume: without supporting material these facts do not travel well; they remain concealed in the image and may go unnoticed by the viewer. The viewer instead may take the image to be an expression of other 'facts', such as that of the power of IBM. However, once the message about the underlying facts has been received, the image alone will suffice for viewers to recall the encapsulated procedural and technological facts. ${ }^{9}$ The careful crafting of the image according to established "macroscopic

\footnotetext{
${ }^{9}$ Contrast this with the case of the silhouettes of raptors that are put on windowpanes to keep birds from flying into windows, a measure which has no scientific backing (Burkhardt, this volume Simona, please supply full ref).
} 
viewing conventions ${ }^{10 "}$ (Hennig 2004: 15) helps to render the image accessible and recognizable by a wider public. As a result, the IBM logo image has today become an element of nanotechnology's iconography. Yet, as an icon, the image no longer only stands for procedural and technological ability; it has also come to symbolize nanotechnology's expected potential and the scientists' power over nature.

\subsection{Scientific Communication}

Scientists acknowledge that the single IBM logo image is a carrier of procedural and technological facts. When I asked a physicist what the image represented to him, he asserted that "it shows the capacity of the researchers to control the position of atoms that they can place without mistake". But how was this image first introduced into the scientific community? It was published in 1990 by IBM researchers Eigler and Schweizer in a three-page letter with the title "Positioning single atoms with a scanning tunnelling microscope" in the journal Nature. With its claim and demonstration that the STM can be used to position individual atoms on a surface with atomic precision, the article raised considerable interest in the scientific community. ${ }^{11}$

When comparing how the 'public' IBM logo image and the visual displays in the Nature article talk to their respective audiences, a number of differences come to the fore. First, it is not surprising that the scientific article contains a wealth of detailed textual information about the experimental process that supports the central claim since, as a general rule, images in scientific articles are always embedded in other types of material.

\footnotetext{
${ }^{10}$ My translation.

${ }^{11}$ In the words of the logo's scientific creators: "This capacity has allowed us to fabricate rudimentary structures of our design, atom by atom" (Eigler and Schweizer 1990: 524).
} 
Second, the scientific article does not exhibit a single (isolated) image of xenon atoms but instead presents a composition of six adjacent images that come in two columns of three images each (fig. 2).

\section{Figure 2}
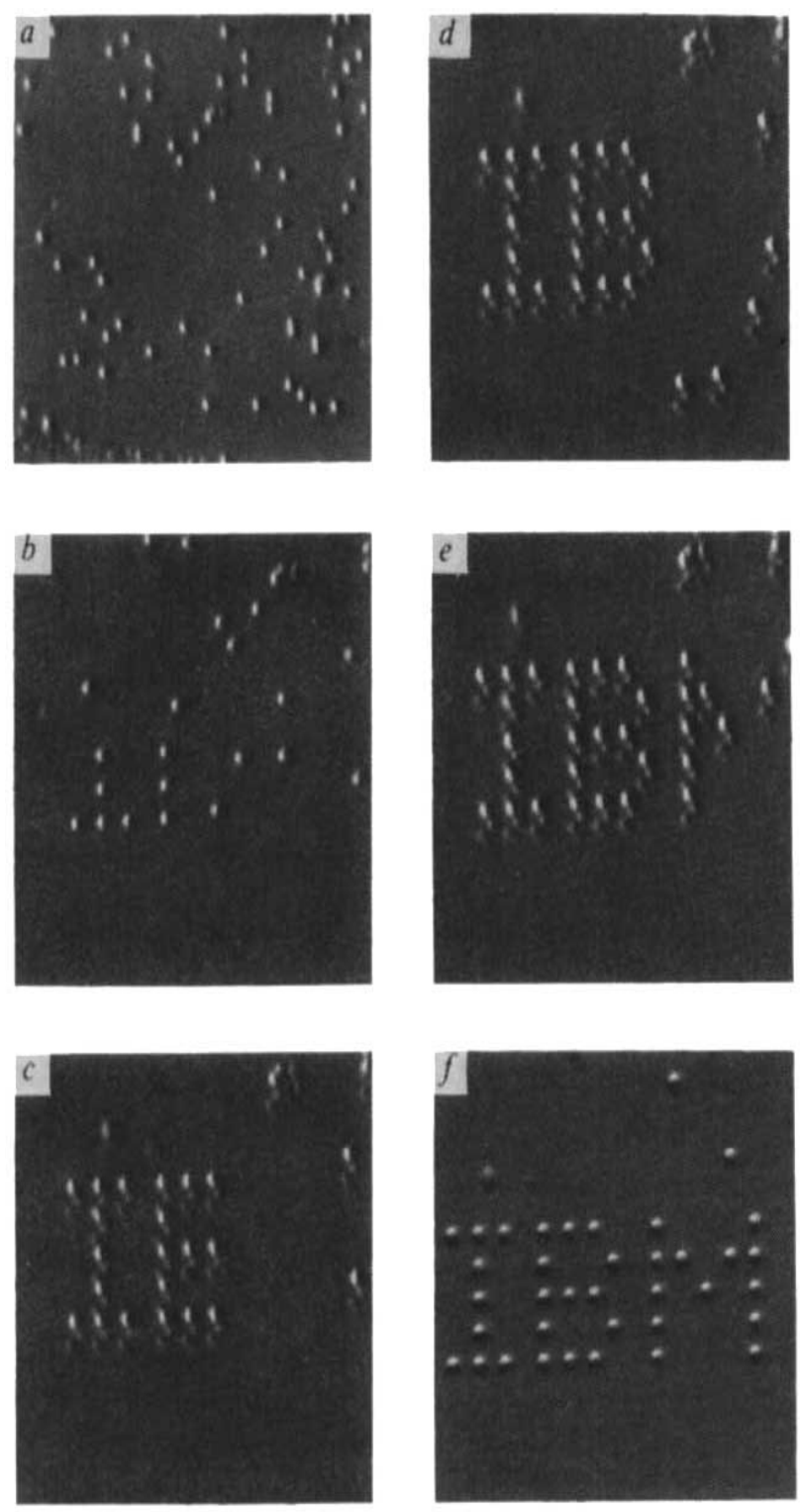

"A sequence of STM images taken during the construction of a pattern array of xenon atoms on a nickel (110) surface. Grey scale is assigned according to the slope of the surface. The atomic structure of the nickel surface is not resolved. The $<110>$ direction runs vertically. a, The surface after xenon dosing. b-f , various stages during the construction. 
Each letter is $50 \AA$ from top to bottom" (legend and figure as in Eigler and Schweizer 1990: 525).

Reprinted by permission from Macmillan Publishers Ltd: Nature (Eigler, D.M. and E.K. Schweizer (1990), 'Positioning single atoms with a scanning tunnelling microscope', Nature 344 (5 April): 524-526), copyright 1990, http://www.nature.com/nature).

The six images exhibit a temporal "sequence of STM images taken during the construction of a patterned array of xenon atoms on a nickel (110) surface" (legend, Eigler and Schweizer 1990: 525), which shows successive stages of the construction process. The composite visual display reinforces the impression of procedure and process: it visually documents and demonstrates the fact that the IBM-pattern can be produced, step by step. In the body of the text, the figure is introduced as "a sequence of images taken during our first construction of a patterned array of atoms, and demonstrates our ability to position atoms with atomic precision" (ibid.). This first figure ${ }^{12}$ is accompanied by two other figures. The second consists of a schematic rendering of how the microscopy tip attracts an atom and moves it across the surface. The third is composed of another block of six images, which uses an alternative form of representation to show "various stages in construction of a linear chain of xenon atoms on the nickel (119) surface" (legend, ibid. 526). All three figures make a factual statement about procedure by explicitly exhibiting the ability of the researchers to position atoms - in contrast, the public IBM logo image conveys the procedural fact in a more implicit manner. While not easy to decipher from the images, the fact that the atomic structure had been both produced and visualized by an STM (technological fact) was mentioned right at the beginning of the legend. After all, this is what made the publication so noteworthy. Concerning the explicit rendering of the temporal dynamics one may wonder whether the Nature article is an exceptional case due to its declared aim to establish

\footnotetext{
12 The first figure of the Nature article corresponds to Figure 2 in this text.
} 
the success of a novel procedure. It will be argued below that procedural facts, which are characteristic outcomes of nanotechnology research, are typically represented in the form of microscopy images embedded in composite visual displays.

A third difference concerns the visual characteristics of the images. The public version of the IBM logo image (fig. 1) turns out to be not simply the last image in the sequence of six (fig.2) shown in the scientific article. Instead, it consists of a careful reconstruction and redesign that shares primarily the abstracted IBM-pattern of the individual atoms with its scientific counterpart(s). In the Nature article the images come in black and white, they are a little blurry, and the contrast between background and signal isn't optimal. Close inspection reveals that in three of the sequence's six images the atoms appear double, due to the STM tip having been "dirty" (Hennig 2009). In contrast, the public image of the single-atom IBM logo seems polished, shiny and colourful. The representation of the atoms as illuminated blobs with shadows is the result of an adaptation to macroscopic viewing conventions (ibid.). This distinction of image design according to scientific conventions and according to the preferences of public media points to a more general trend, caught tellingly in the opposition of a "rhetoric of rough" for the case of science and a "semiotics of smooth" as illustrated by the public IBM logo image (Curtis 2007). Of course, design conventions of probe microscopy images (and other kinds of images) are today heavily debated in the scientific community. Also whilst researchers follow the trend toward more sophisticated and colourful renderings they insist, at the same time, that images deemed good according to scientific standards may (need to) look "dirty" to less experienced people.

The assessment of the STM-imagery as it appears in the Nature article suggests tentative conclusions, which may serve as hypotheses for the further investigation of facts that travel in images within a scientific 
community. First, STM-images emphasize procedural capacity (procedural fact) while other types of facts are communicated less explicitly. Second, an image rarely comes alone; it is typically accompanied by other images and visual representations. The question then arises how these features relate to how well facts travel in scientific publications.

\section{Images in Scientific Articles}

As a central medium of scientific communication, the research article in the sciences cannot be imagined without the presence of images, diagrams, tables, graphs, and other types of visual representations. The science studies literature ${ }^{13}$ has addressed the scientists' production, transformation and diffusion of visual representations with an emphasis on image multiplicity, aptly caught by Bruno Latour's pithy phrase:

An isolated scientific image is meaningless, it proves nothing, says nothing, shows nothing, has no referent. (Latour 2002: 34)

Image multiplicity has been discussed with respect to the production of images in the scientific laboratory (Amann and Knorr Cetina 1990; Lynch 1985 ) and the diffusion of visual representations from the laboratory to the public (Latour 1990). In both cases, the studies drew attention predominantly to "serial" relations of images (Lynch and Woolgar 1990b: 6 ) - that is, the directed transformations of visual representations that render the underlying phenomena of investigation progressively "more

\footnotetext{
${ }^{13}$ The influential collection of articles Representation in Scientific Practice, edited by Lynch and Woolgar (1990a), put the analysis of scientific representational practices on the agenda of science studies. For a comprehensive introduction to the analysis of the social practice of scientific imaging and visualization see Burri and Dumit (2008).
} 
visible, stable, and measurable" (ibid.) or, to put it in Latour's terms, the "cascade of ever simplified inscriptions" (Latour 1986: 16). In contrast, "transversal" (i.e. non-sequential) relations between visual representations - as I will call them - have received little attention (Alač 2004; Bastide 1990; Lynch 1990; Myers 1990).

In what follows, the transversal relations of images and other visual displays within a scientific text will be the centre of attention. This will require the exploration of the mutual contextualization of the figurative elements within an article, considering not only the relations of these elements among each other but also the figurative and the textual elements. For this purpose, it seems fruitful to conceive of a visual display as "an autonomous surface that is nonetheless contained within a text" (Lynch 1990: 155). From this perspective, instead of reducing visual representations by default to the role of merely illustrating the text, the relation of visual display and text is open to negotiation. Based on the hypothesis that something interesting is happening to the underlying facts when figurative elements are assembled into composite visual displays, this section will first look at the visual 'fingerprint' of displays and articles. It then recapitulates three types of facts that travel in images, and finally assesses scientists in their roles as readers and writers to learn more about the relation of facts and images.

\subsection{Composite Visual Displays}

When leafing through the pages of journals in the field of nanotechnology it is eye-catching that, as in the case of the aforementioned Nature article, the visual displays - i.e. what is subsumed and bracketed under the label "Figure" in an article - are predominantly composed of several images, curves, and schemas together. In the following, the term "composite visual displays" refers to such compositions that join and gather several images, curves and/or schemas 
within a common frame (visual display), complemented by a joint legend and under the header of a specific figure number. ${ }^{14}$

To get a better grip on the typical form of an article's composition of textual and visual elements, two hundred articles have been assessed numerically. The articles selected for analysis were taken from two journals: the first is the journal Nanotechnology, which is dedicated to covering research in nanoscale science and technology from a multidisciplinary perspective; the second is the journal Advanced Materials, one of the top international materials science journals, read by materials scientists, chemists, physicists and engineers of various orientations and the nanotechnology community. ${ }^{15}$ From each journal one hundred articles were considered, 25 each from 1992, 1993, 2007 and 2008.

Visual material has a very high status in both journals, as measured by the space it occupies in an article: the relation of visuals (including legends) and running text is roughly one to three. Indeed, a microscopy image rarely appears on its own (this happens in only $5 \%$ of articles in $2007 / 08$ ); in about $40 \%$ of the articles several microscopy images are exhibited in direct juxtaposition (47\% in $2007 / 08$ ); in about $30 \%$ of the articles microscopy images are combined with curves (48\% in 2007/08); in about $15 \%$ they are combined with schemas (21\% in 2007/08). Thus, in many cases composite visual displays (which contain an average of about four elements in 1992/93 and seven elements in 2007/08) have a more complex internal referential structure than the sequence of the IBM article.

\footnotetext{
${ }^{14}$ The journal Advanced Materials calls such structures "multi-panel images" but this terminology is not followed here to avoid confusion. The term "image" is reserved for a single visual display, typically in contrast to other types of visual displays such as schemas or curves.

${ }^{15}$ The journal Nanotechnology was founded in 1990 and currently has an ISI Impact Factor of 3.3 (in 2007). The journal Advanced Materials has an ISI Impact Factor of just above 8 (in 2007) and celebrated its $20^{\text {th }}$ Anniversary in August 2008.
} 
This analysis confirms what other sources (e.g. poster presentations, further journals) suggest. First, images are very abundant: today, only one in eight articles contains no microscopy image at all. ${ }^{16}$ Second, an image is rarely presented on its own in scientific publications. This text argues that the companionship of other visual representations ensures that the facts that are embedded in images travel well across the scientific community. The contention is based on the understanding that an image acquires meaning in the context of and when juxtaposed with other images, schemas, curves.

\subsection{Embedding Facts in Images}

As the discussion of the IBM logo image suggests, factual statements of different kinds are embedded in and can be uncovered from individual microscopy images as well as from an ensemble of images. This potential of images to carry various kinds of facts resembles that of material objects, which can "store and communicate" facts as diverse as material, technical, user-related facts, etc. (Valeriani, this volume Simona, please supply full ref). The factual multi-valence of scientific images is related, one may assume, to their "semiotic openness" in combination with "their being regarded as the simultaneous voice of technoscientific authority and as expressions of nature" (Burri and Dumit 2008: 305). This characteristic might account for the willingness of readers to assign fact-status to images while the specific kind of factual statement is co-determined by the image's context. The factual statements are about (at least) three kinds of entity:

- Phenomenon: Microscopy images "reveal" (in the scientists' terminology) that the underlying phenomenon or object of

\footnotetext{
${ }^{16}$ The reasons for the abundance of visual displays in scientific articles cannot be discussed in detail here but one may assume that the progress of image reproduction technologies and the reduced cost of image production play an important role.
} 
investigation has a certain shape. In the case of probe and electron microscopy this is typically a factual statement about the atomic or molecular structure of the sample of interest.

- Procedure/process: The prime factual statement that is communicated by way of an image (or visual display) does not necessarily concern the rendered phenomenon, respectively its atomic structure. Instead, the image can highlight more specifically that the exhibited features are the result of a certain experimental procedure or process. The image of the IBM logo, for example, conveys the fact that individual atoms can be deliberately positioned on a surface in a selected pattern by following a certain procedure, detailed in the accompanying text. The fact that this exercise was conducted with a specific kind of atoms and a specific choice of surface is of lesser importance, although it is of course relevant to fellow scientists who might want to replicate the experiment.

- Technology: In other cases, the communicated factual statement primarily relates to the technology used to produce what the image reveals. It is a statement about technological capacity and might. This concerns not only the specific form of microscopy used but also other kinds of visualization technology, such as image analysis software, whose importance for the production of the published images should not be underestimated. Factual statements about technology are specifically important in periods in which a technology becomes newly established. In these cases, the focus shifts from the portrayed (phenomenon) to the portraying instrument: the images may reveal the capacity of the underlying technology, while the imaged phenomena assume an instrumental role. Probe microscopy (such as STM, AFM) has been established over the last two decades (Mody 2004). As a consequence, probe 
microscopy images are today no longer associated with the underlying technological facts to the same degree as they used to be.

Thus, microscopy images can carry various kinds of factual statements. These may be statements about the visualized phenomenon, about the procedure and process followed to bring about the phenomenon or about the underlying technology. An image can be but does not need to be associated with one specific factual statement - there is no one-to-one correspondence between image and fact. An image may also carry various kinds of statements to different degrees. An example is once again the IBM logo image, which reveals process and technology alike. This is a typical feature of probe microscopy, as it is used both to visualize and to produce the imaged atomic structure, which leads to a tight coupling of instrument and production procedure.

Typically, the image itself does not determine the underlying factual statement. The kind of factual statement is rather assigned through the interaction with the image environment, that is, with the other visual displays and the texts around it. In a way then, it is an emergent property. As a result, facts are not only embedded in (and emerge from) individual images but also in composite visual displays.

\subsection{Visual Narratives: "By Looking at the Figures I Should get the} Story"

A research article attracts the attention of peers not only because of its scientific quality and innovative character. Visibility depends decisively also on the reputation of the scientific journal in which the article appears and on how the results are 'packaged'. How scientists select and present images and visual displays in their publication thus plays an important role for producing visibility for their results. Scientists are confronted with this issue from two complementary perspectives: as authors and as 
readers. They have expertise both in packaging facts for travel in the form of images and visual displays and in extracting them from the article with its textual and visual material as readers, these two forms of expertise feeding on each other.

How then do scientists acquaint themselves with the scientific literature in their field of expertise ${ }^{17}$ In a first step, they identify publications of potential interest according to an article's title, authors and abstract and then download the articles for closer scrutiny. In a next step, the readers turn to the images and their legends: ${ }^{18}$

"I read the abstract, I look at the pictures and the legend that's what I do first. And then it depends, whether I quickly scan through the article, how important it is." (Interview)

The reader's move from images and legends toward the surrounding main text has a correspondence in how scientists write an article. The first step consists of putting the figures together, the next "to write the article around the figures" (interview). Authors add the legends first and then, step by step, the remainder of the text. The preposition "around" suggests that the visual displays are conceived of as the article's centre and core. The centrality of the figurative characterizes the article as it determines both the reader's and the author's focus of attention.

But how should this visual centre be considered? As the assessment in section 3.1 suggests, the scientists group images and visual representations in composite visual displays, resulting in only a few figures per article. The reason scientists give for not exhibiting individual

\footnotetext{
${ }^{17}$ The following is based on qualitative interviews with senior scientists in the field of nanoscale science.

${ }^{18}$ In his seminal work on the genre of the experimental article, Bazerman (1988, chap. 8 ) also discusses how physicists read physics literature. The insignificance of visual displays in this case - Bazerman mentions physicists "perhaps scanning figures" (ibid. 243) only in passing - reminds us that visual displays were not of central importance to all physics specialties at that time.
} 
images is that this "makes the story very hard to read" (interview). Instead, "putting information together" (interview) in the form of composite visual displays is seen as a way to package related facts into a "local story", which ensures that the facts become optimally accessible to the readers (cf. section 4 ).

What do the researchers mean when they say that a visual display tells a story? One may suppose that the scientists' stories, like narratives, "create a sense of why things happen" (Hayles 1999: 10). Such stories emphasise, among other things, temporal sequence and causality (ibid.; cf. also Bruner 1990). Visual displays and their individual components provide accounts of the underlying processes (temporal sequence) and they allow the viewers to construct an account of why the represented events occur (causality). Stories embedded in visual displays involve two kinds of causes: on the one hand, the scientists' motivations and procedures are the cause of a certain experimental course of action, which an article's readers attempt to decipher from the visual displays; on the other hand, the outcome of experiments is interpreted as having certain natural causes, which refers back to phenomenal facts and their interpretations. These two kinds of causes are entangled in the stories that scientists uncover from visual displays.

Consider once more fig. 2 , the composite visual display that shows how the IBM logo image comes into being. The story embedded in the figure simultaneously emphasizes temporal sequence and causality: the sequence of STM images presenting the deliberate and successful act of the researchers to produce the I-B-M pattern of individual atoms step by step. Such stories are a way to communicate different kinds of facts. While the story associated with the IBM figure highlights a procedural fact, a story may also evolve around facts about phenomena and/or technology. In order to allow readers to construct a story, a visual display has to exhibit a certain complexity and, typically, a composite nature. In 
many cases, this involves (in contrast to fig. 2) that a figure contains different forms of visual representation, such as a combination of images and schemas. It should be noted, though, that stories that materialize around visual displays typically do not have the densely layered texture that is characteristic of fictional narratives. ${ }^{19}$ Perhaps surprisingly, this feature does not seem to be necessary to create a sense of why things are happening in an experimental setting.

Clustering visual representations in the form of composite visual displays means that the spatial relationship between the visual representation and its associated referring text is broken up - after all, each figure corresponds to a precise passage in the text that refers to it (Myers 1990: 249). The distance between image and referring text is yet another indication for the high degree of autonomy of the narrative that is created by visual representations and their legends within a composite visual display. Despite the autonomy that engenders "local stories" (as the interviewee put it), there is a sharp awareness of the correlation between the different visual displays in one article and the requirement that they sum up the main content and results of the paper. This requirement is summarized by the advice a senior scientist gives to his student:
"By looking at the figures I should get the story. If the figures are not telling me the story, you are missing one or two. Or you didn't choose them properly." (Interview)

The quote implies that the visual displays together should be comprehensive and represent all central moves and outcomes of the article. From this perspective, the main text can be interpreted as an

\footnotetext{
${ }^{19}$ On the role of narrative in helping facts to travel see Adams (this volume Simona, please supply full ref).
} 
extended legend to the figures, supplemented by information about the motivation of the work and how it relates to similar work.

\section{Communicating Facts in Composite Visual Displays}

In what follows, a specific composite visual display will be examined more closely from two contrasting perspectives: first, the authors' perspective as reconstructed from the article in which the figure appears (4.1) and, second, the perspective of a reader (4.2). I had asked senior scientist Barbara ${ }^{20}$ - a physicist by training who has worked in nanoscale science for many years - to select articles that she planned to read. One of them is the article that will feature here. The publication is authored by a group of scientists from Beijing and recently appeared in the prestigious journal Physical Review Letters under the title "Probing Superexchange Interaction in Molecular Magnets by Spin-Flip Spectroscopy and Microscopy" (Chen et al. 2008).

\subsection{Zoom in on a Composite Visual Display}

The article's main running text is combined with four composite visual displays and one table. Of the four pages about 1.5 pages are covered with figures and their legends. The two columns of page two are each half filled with a colour figure ${ }^{21}$ : FIG. 1 (corresponding to fig. 3 below) presents a combination of microscopy images and schematic representations; FIG. 2 exhibits four graphs arranged in the form of a square. On page three, FIG. 3 joins three graphs and two schematic renderings. On the last page, FIG. 4 combines two schemas, three measurement curves with a joint heading, a superposition of an image

\footnotetext{
20 This is a pseudonym.

${ }^{21}$ To avoid confusion, the figure numbers are labelled in capitals when the numbering of Chen et al. (2008) is concerned and in lower case when this article's numbering is referred to.
} 
and a schema, and, finally, a visual rendering of a calculated structure. The article is therefore a good example of the present trend toward more complex composite visual displays.

The four figures seem to segment the paper and align the fourteen paragraphs, which are not subdivided into sections. After the first two paragraphs, which introduce the reader to the topic, provide a motivation and present an outline of the article, two to three subsequent paragraphs each are associated consecutively with each of the four figures: they introduce the molecular structure (FIG. 1), the results of scanning tunnelling spectroscopy measurements (FIG. 2), the spin-flip spectra (FIG. 3), and the superexchange mechanism (FIG. 4). The article closes with an outlook and acknowledgements, followed by the list of references.

Let us take a closer look at the first figure. It is the only one dominated by microscopy images and it is also the one that attracts reader Barbara's attention (cf. 4.2). The figure is visually structured by two types of elements: the three annotated STM images (a, c, d) on the one hand, the two schemas in the upper right corner (b) and at the figure's bottom (e) on the other hand. The three images seem to form a unit, an impression that is reinforced by the same colours being used; the two schemas appear to constitute a frame to this image block. Upon consideration of the composition and the content of the visual representations, there does not seem to be a clear entry point into the figure. The detailed representations of (c) and (d) are unlikely to constitute a starting point and nor is the complex rendering of schema (e) with its two inserts, but both (a) and (b) look like probable candidates and it will be shown that they are.

Insight into the authors' perspective on the composite can be gained by considering the alphabetic order of its visual representations, which suggests a reading order, and by unravelling the figure's legend and the corresponding running text. 
Image (a): FIG. 1(a) presents an overview of the multilayered molecular structure of interest. The legend introduces it as an STM image of "self-assembled multilayers of CoPc molecules" 22 that sit on a lead surface. The statement emphasizes both the imaging technology and that the structure is lab-produced. Indeed the procedure is described in the running text for readers interested in the details: "Cobalt phthalocyanine $(\mathrm{CoPc})$ molecules were then thermally sublimed onto the $\mathrm{Pb}$ islands at room temperature to form a self-assembled monolayer with square lattice pattern. Subsequent sublimation of CoPc was performed at sample temperature of $\sim 120 \mathrm{~K}$ to form the ordered multilayer structures, as shown in Fig. 1(a)" (p. 1). The running text clearly labels image (a) as associated with the procedural fact by referring to it at this precise spot. The image also gives rise to the appreciation that it had been produced by STM (technological fact), the details of which (voltage and current) are presented in the legend.

${ }^{22}$ This and the following quotations refer to Chen et al. (2008) unless otherwise indicated. 
Figure 3

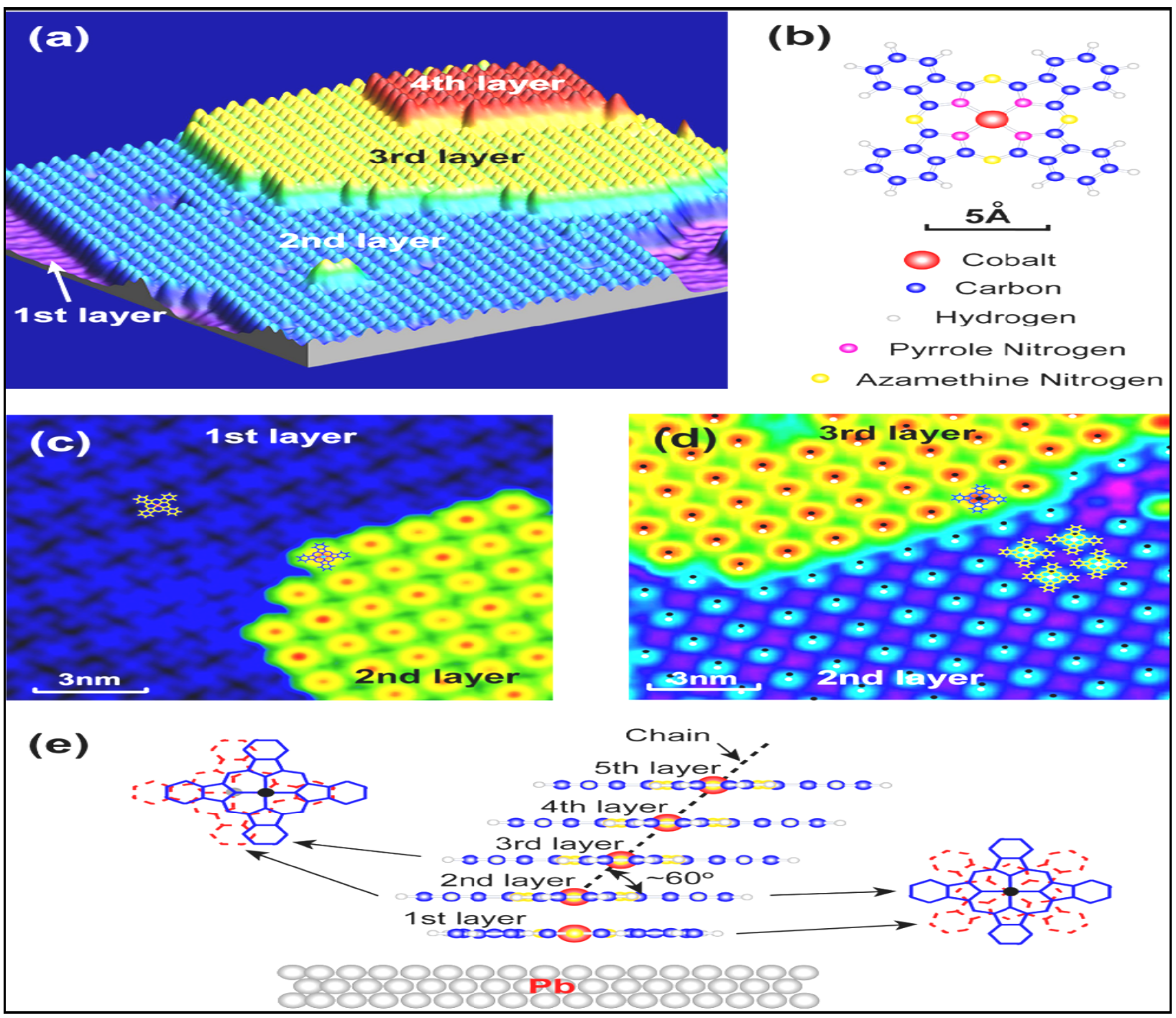

"FIG. 1 (color online). CoPc multilayers on Pb. (a) STM image $(\mathrm{V}=0.9 \mathrm{~V}, \mathrm{I}=0.03 \mathrm{nA}$ ) of the self-assembled multilayers of CoPc molecules on $\mathrm{Pb}(111)$ film (26 ML thick). (b) Molecular structure of CoPc. (c) STM image $(\mathrm{V}=0.6 \mathrm{~V}, \mathrm{I}=0.1 \mathrm{~A})$ showing the relative stacking of the $1 \mathrm{st}$ and 2nd CoPc layers. (d) STM image showing the relative stacking of the 2nd and 3rd CoPc layers. The white and black dots indicate the centres of the molecules on the 2nd and 3rd layers, respectively. (e) Stacking geometry of CoPc molecules. The molecular layers are spaced $3.5 \pm 0.1 \AA$ apart. The inserts show the orientation and displacement between molecules in adjacent layers."

(Legend and figure as in Chen et al. 2008: 2, Reprinted with permission from Chen, Xi et al., Physical Review Letters 101 (19), Fig. 1, 2008, Copyright (2008) by the American Physical Society) ${ }^{23}$

\footnotetext{
${ }^{23}$ Readers may view, browse, and /or download material for temporary copying purposes only, provided these uses are for non-commercial personal purposes. Except as provided for by law, this material may not be further reproduced, distributed, transmitted, modified, adapted, performed, displayed, published, or sold in whole or part, without prior written permission from the American Physical Society http://publish.aps.org
} 
Schema (b): The schema depicts the molecular structure of one such Cobalt phthalocyanine molecule and thus stands in for a phenomenal fact. It presents a full figure within the figure as it has its own legend - explanations of the colour codes of the chemical elements, which make up the molecule - and also comes with a scale (5 $\AA$ ), which makes it stand on its own. Accordingly, the running text adds little further information.

Images (c) and (d): The next two "zoom-in STS images" are presented as a package (albeit with individual numbers) as they come with the same size, scale, and orientation. They show details of the "stacking geometry of CoPc in the multilayer structures" with a focus on the $1^{\text {st }}$ and $2^{\text {nd }}$ layers (c) and the $2^{\text {nd }}$ and $3^{\text {rd }}$ layers (d). As zoom-ins they are directly associated with STM-image (a) of which they present a more detailed and fine-grained picture, which is further detailed in the running text, but they are also intimately connected with schema (e). They show how a layer is positioned with respect to the next one and how the molecules are arranged (phenomenal fact).

Schema (e): The schema provides a model of how the different layers sit on top of each other, indicating the orientations and displacements of the molecules in differing layers. Inserts, linked up by arrows with two layers each, show in more detail how molecules that sit on top of each other are twisted against each other. The schema summarizes the relevant features of the stacking geometry, drawing heavily on information from the STM-images. As such it constitutes an "image of synthesis" (Allamel-Raffin 2006). What about the factual status of this schematic representation? The corresponding caption leaves no doubt, it represents the structure (phenomenal fact), associated with a 
determined "stacking geometry" and a quantified spacing between molecular levels.

The construction logic underlying the composite visual display suggests to move from FIG. 1(a), which specifies the technology used and the procedure followed to generate the multilayers of CoPc molecules, to FIG.s 1(b), (c), and (d), which provide information about the phenomena, i.e. the stacking geometry of these multilayer structures, and finally to FIG. 1(e), which synthesizes the phenomenal statements. While facts about procedure and technology are conveyed by the figure and its legend, the emphasis of this composite is on the phenomena - at least, this is the impression gained by reconstructing the authors' perspective. In the next section, a reader in an attempt to make sense of it approaches the same composite figure.

\subsection{A Reader's Perspective}

Only readers can provide the ultimate proof that facts are communicated through images in scientific articles. Readers can show how successful they are in unpacking visual displays to access the underlying facts. For this reason, it seemed fruitful to observe how scientists read a scholarly publication, how they attempt to extract facts from the imagery and how they reason in this process. To make reading observable it, above all, has to be made audible. ${ }^{24}$ Hence, I had asked senior scientist Barbara to go through the articles she had previously selected for closer inspection audibly, letting me observe how she made sense of the articles and their visual displays. Her initial monologue, followed by a few questions of mine requesting further specification, was recorded and transcribed and presents the empirical material of this section.

\footnotetext{
${ }^{24}$ For a discussion of how thinking and other allegedly 'immaterial' practices can be observed cf. Knorr Cetina and Merz (1997).
} 
Barbara had selected the article by Chen et al. (2008) on the basis of its title and abstract. She takes the article, runs over the pages and flips it open at page two, turning to FIG. 1 (fig. 3 here) and FIG. 2 that exhibits four graphs. During the seven minutes that we spend on the article, her eyes and fingers move back and forth between the individual components of the first figure and between the two adjacent figures. The movements would have created an interesting zigzag pattern when recorded visually. ${ }^{25}$ With some limitation, this zigzagging movement becomes traceable also when looking at the transcript.

She starts to muse about what the figures show by addressing the article's first figure in the following way:

"First, what is important, what is all this about, which molecule on which surface? Here (FIG. 1b) I see the molecule, then I read the caption, on lead, so it's on lead 1-1-1, then I look at the corresponding image (FIG. 1a), and then I already see, okay, in this case we are dealing with multilayer growth, so $2^{\text {nd }}$, $3^{\text {rd }}, 4^{\text {th }}$ layer. This is STM and it is turned. All right, and next: here is again schematically how it looks like (FIG. 1e). What do I look at next? Then I see the resolution is good (FIG. 1c-d), here you see single molecules, now these are phthalocyanines." [Interview transcript 01.09.22]

The scientist does not pause at any one image or schema but creates a narrative that smoothly moves from one element to the next. Considering the statements in turn allows one, once again, to associate them with the different types of facts.

Schema (b) and legend: Barbara's opening question "what is all this about?" inquires into the specific sample that was investigated. An answer is provided by a combination of the schematic representation, which shows the chemical structure and composition of the concerned

\footnotetext{
${ }^{25}$ Ideally, the observation of scientists making sense of visual displays should be video-recorded.
} 
molecule, and the legend, which informs her that these molecules sit on a lead surface. Although the composite visual display is visually dominated by three microscopy images, Barbara's first gaze is directed at a schema - which in this instance provides a first insight into the structure of the phenomena under investigation (phenomenal fact).

Image (a): She then turns her attention from the schema toward the large microscopy image (a), which shows that the molecules do not cover the lead surface uniformly but come in layers, four layers being clearly visible as each layer has a different colour and is annotated correspondingly. This image, on the one hand, provides further insight into the phenomena under investigation (phenomenal fact). On the other hand, Barbara interprets it as hinting at the underlying manufacturing process (procedural fact), which she addresses by the notion "multilayer growth" - the layers do not sit naturally on the lead surface, they have to "grow", which requires a dedicated technical procedure. Barbara also acknowledges that an STM was used to visualize the structure (technological fact).

Schema (e): The next visual representation in focus is once again a schema, the second in the display, which Barbara scans only quickly with the words "here is again schematically how it looks like" (phenomenal fact).

Images (c) and (d): Barbara then turns to the two smaller images but instead of scrutinizing the details of the molecules' stacking configurations, she is merely interested in the images' resolution, which she identifies as good because single molecules are visible. This fact about the imaging arrangement (technological fact) provides trust in the experiment as a whole. Technological facts are not only associated with the capacity of technologies but also with the skills and precision of the scientists that handle them, as this case illustrates. The statement with which Barbara closes her consideration of the entire figure "now these are 
phthalocyanines" seems to draw all components of the composite together. With this statement she also comes full circle with her initial question "what is all this about, which molecule on which surface?".

Of course, this is not the entire story. The article contains another three figures and Barbara's account on the article's visual displays is more extended, while she does show most interest in FIG. 1. The following considerations will use the above for illustration but take other cases into account as well. This will allow me to draw out some specifics of how facts are communicated through images and visual displays.

\subsection{Observations}

An image contains indications about different kinds of facts: a microscopy image may provide factual information about phenomena, procedure and technology alike. Whether one of these types of facts will emerge as a dominant feature will depend on the image's context as much as on the reader's specific expertise and interest. For example, the legend may suggest the type of fact to be communicated by an image. Alternatively, an entire article may be explicitly associated with one type of fact. An example is the Nature article that displays the IBM logo images to communicate the procedural fact of this type of structure's fabrication.

The explored cases show that a specific type of fact is rarely communicated in isolation by an image. Factual statements about procedure and technology are closely associated (e.g. fig 2), an image that exhibits a phenomenal fact may also communicate a procedural fact (e.g. FIG 1a), etc. It seems that scientists have a preference for communicating loose fact bundles of this kind. This may explain their penchant for composite visual displays, which create an environment in which facts about procedure, phenomena and technology circulate and are tied together. How Barbara traces the individual components of the figure to make sense out of it is a telling illustration: phenomenal fact ( $b$ 
and legend) $\rightarrow$ phenomenal fact and procedural fact $(\mathrm{a}) \rightarrow$ technological fact (a) $\rightarrow$ phenomenal fact (b) $\rightarrow$ technological fact $(c, d) \rightarrow$ summary of entire figure.

When comparing the (reconstructed) authors' and readers' accounts of the composite visual display FIG. 1, one first notes, importantly, that there is no indication that (major) facts are lost on the way between author and reader. However, authors and readers tie visual representations and facts together in a different manner - and it can be assumed that there is a wide variety of ways to do so on both the authors' and the readers' side. One reason for this heterogeneity is that both address the visual displays on the basis of their respective background knowledge and purposes (Bazerman 1988, chap. 8). Consider the reading order. A composite visual display comes with a reading order suggested by its authors. In the discussed case it moves through the visual representations of FIG. 1 sequentially, from (a) to (e), communicating fist procedure and technology, then phenomenal facts with increasing detail and breadth, ending with an image of synthesis (cf. 4.1). Reader Barbara does not follow this order but selects her own, based on her personal interest, experience and knowledge: she zooms in on the schematic representation (FIG. 1b) of the specific molecule first because she is curious about the particular "magnetic atom in such an organic molecule" and what one might be able to do with it. The multilayer structure of such molecules (FIG. 1a) is of interest to her in the next step. The composite visual display allows readers to be flexible in the reading order they choose. The spatial composition of the display - typically visual representations are not aligned but rather grouped loosely within a rectangular frame - invites readers to choose their own passageway through the display to make sense of it.

Composite visual displays provide flexible fact retrieval mechanisms not only because they allow viewers to determine their own 
reading order. They are characterized also by multiple cross-referencing of their individual components, each acquiring meaning and fact status in view of the others (Alač 2004; Bastide 1990), which helps the facts to circulate within the composite. Cross-referencing employs a variety of reference forms: e.g. comparison with an alternative (sample, instrumental representation, visual representation, etc.), zoom (i.e. change of scale), abstraction, concretization (e.g. image versus schema), etc. The discussed case of FIG. 1, for example, contains referencing relationships of zoom (a versus $c$ and d), of abstraction ( $a, c$, and d versus $e$ ) and of comparison (c versus d). Such transversal relations and mutual contextualizations between individual visual representations in a composite display assist viewers in constructing a story by associating the circulating facts.

A composite presents an assembly of different kinds of facts and cross-reference associations between the different visual representations. The reader fits these elements together to create a sense of what happens in the underlying experiment and why things happen. Also the legend assists this endeavour, acting as supplement and intermediary between the components of a complex visual display. It fills in what remains open in the visual material. For example, in the case at hand, it indicates that the substrate consists of lead on top of which the molecule layers are deposited, as the images show. It also provides the parameters of the STM, and it offers assistance with interpreting the white and black dots in the microscopy images. The legend also provides a link between the display and the main running text. As such, it enables the facts to be tied to their qualifiers and to contextual information about their origin, their experimental underpinnings and their expected value.

The question of how well facts travel in images and composite visual displays within a scientific community is thus less one of facts 
getting lost or being misinterpreted. ${ }^{26}$ It is rather a question of whether flexible fact retrieval mechanisms such as composite visual displays exist that enable scientists at the receiving end to construe a rich story of phenomenal, procedural and technological facts, full of dynamics and hints at the underlying experimental practice.

\section{Conclusions}

This text has attempted to show how microscopy images in the nanometre range can be effective devices to communicate facts. Yet, it has also argued that facts do not automatically travel when images are diffused. ${ }^{27}$ Above all this is the case when images travel from science to the public. The case of the IBM logo image illustrated that the image may lose its (procedural and technological) facts when being diffused to nonexpert communities who had not been exposed to the image previously. Thus, the facts did not travel well in the sense that viewers could not easily unravel them. To travel well, these facts need to be packaged more thoroughly with legends and explanations that accompany the image. To travel well they rely on a certain degree of scientific understanding on the viewers' side. This seems to be a typical feature of communicating facts by images. The semiotic openness of images (cf. 3.2) implies, on the one hand, that they are ruled by requisites (e.g. prior knowledge and experience) while they are, on the other hand, very suggestive and draw their force from association with visual conventions. This is why the facts embedded in the IBM logo image are easy to recall once the main message has been received but remain buried otherwise.

\footnotetext{
${ }^{26}$ The travelling of facts within an expert community is the concern of several chapters in this volume; see, for example, the contributions by Leonelli, Valeriani, and Whatmore and Landström. (Simona, please supply full ref)

${ }^{27}$ Schneider (this volume) makes the same point in the context of architecture.
} 
The communication of facts through images follows other rules within the scientific community, mainly because the viewer's preconditions are different. Members of a scientific community share visual conventions, expertise concerning how to produce, edit and interpret images, etc. They are skilled both in writing and reading scientific publications, which implies that they can swap perspectives. These are skills that distinguish scientists from public viewers. When comparing how facts are communicated by images to the public and within a scientific community, the most striking difference is that a single image is rarely used to transmit facts. It is as if scientists need more than one image to be convinced. In addition, a microscopy image typically allows readers to uncover more than one kind of fact. These two features combined enable a complex visual transmission of facts in research articles. Microscopy images are typically embedded in composite visual displays, the strength of which is that they embed factual statements that not only refer to the phenomena but also to the followed procedure and the adopted technology. One reason why facts travel well in such arrangements is that they allow scientists to construct narratives about the underlying experiment: they tell a story (or allow readers to tell a story) about procedures, technologies, and the structure and behaviour of phenomena all at once. This introduces a temporal order into the interpretation of visuals: the spatial relations within visual displays can thus be transformed into temporal ones. This move inverts the process that Rheinberger (2006: 352) describes as typical for laboratory practice: to convert the spatiotemporal arrangement of a lab into a two-dimensional frame through a variety of notation and inscription practices. While the temporal order uncovered from visual displays is a deeply reconstructed one, which does not provide a faithful account of an experiment's history, it still provides scientists with a useful guideline for how to unravel facts from visual displays. 


\section{Bibliography}

Alač, Morana (2004), 'Negotiating Pictures of Numbers', Social Epistemology 18 (2-3): 199-214.

Allamel-Raffin, Catherine (2006), 'La complexité des images scientifiques:

Ce que la sémiotique de l'image nous apprend sur l'objectivité scientifique', communication \& langages 149: 97-111.

Amann, Klaus and Karin Knorr Cetina (1990), 'The fixation of (visual) evidence' in Michael Lynch and Steve Woolgar (eds.), Representation in Scientific Practice. Cambridge MA: MIT Press, 85-121.

Baird, Davis and Ashley Shew (2004), 'Probing the History of Scanning Tunneling Microscopy' in Davis Baird, Alfred Nordmann and Joachim Schummer (eds.), Discovering the Nanoscale. Amsterdam: IOS Press, 145-156.

Baird, Davis, Alfred Nordmann, and Joachim Schummer (eds.) (2004), Discovering the Nanoscale. Amsterdam: IOS Press.

Bastide, Françoise (1990), 'The iconography of scientific texts: principles of analysis' (translated by Greg Myers) in Michael Lynch and Steve Woolgar (eds.), Representation in Scientific Practice. Cambridge MA: MIT Press, 187-229.

Bazerman, Charles (1988), Shaping Written Knowledge: The Genre and Activity of the Experimental Article in Science. Madison, Wisconsin: University of Wisconsin Press.

Binnig, Gerd et al. (1982), 'Surface Studies by Scanning Tunneling Microscopy', Physical Review Letters 49 (1): 57-60.

Bruner, Jerome (1990), Acts of Meaning. Cambridge MA: Harvard University Press.

Burri, Regula Valérie and Joseph Dumit (2008), 'Social studies of scientific imaging and visualization' in Edward J. Hackett et al. 
(eds.), The Handbook of Science and Technology Studies. Cambridge MA: MIT Press, 297-317.

Chen, Xi et al. (2008), 'Probing Superexchange Interaction in Molecular Magnets by Spin-Flip Spectroscopy and Microscopy', Physical Review Letters 101 (19): 197208 (4 pages).

Curtis, Scott (2007), 'The Rhetoric of the (Moving) Nano Image'. Talk presented at the Workshop Images of the Nanoscale: From Creation to Consumption. NanoCenter, University of SouthCarolina, Columbia SC. October 2007.

Daston, Lorraine and Peter Galison (2007), Objectivity. New York: Zone Books.

Eigler, D.M. and E.K. Schweizer (1990), 'Positioning single atoms with a scanning tunnelling microscope', Nature 344 (5 April): 524-526.

Hayles, N. Katherine (1999), 'Simulating narratives: what virtual creatures can teach us', Critical Inquiry 26 (1): 1-26.

Hennig, Jochen (2004), 'Vom Experiment zur Utopie: Bilder in der Nanotechnologie', Bildwelten des Wissens. Kunsthistorisches Jahrbuch für Bildkritik (Berlin: Akademie Verlag) 2 (2): 9-18.

Hennig, Jochen (2006), 'Changes in the design of scanning tunneling microscopic images from 1980 to 1990' in Joachim Schummer and Davis Baird (eds.), Nanotechnology Challenges: Implications for Philosophy, Ethics and Society. Singapore et al.: World Scientific Publishing, 143-163.

Hennig, Jochen (2009), Bildpraxis: Visuelle Strategien in der frühen Nanotechnologie. Bielefeld: transcript, forthcoming.

Knorr Cetina, Karin and Martina Merz (1997), 'Floundering or Frolicking How does Ethnography Fare in Theoretical Physics? (And what Sort of Ethnography?)', Social Studies of Science 27 (1): 123-131. 
Landau, Jamie et al. (2009), 'Visualizing Nanotechnology: The Impact of Visual Images on Lay American Audience Associations with Nanotechnology', Public Understanding of Science 18 (3): 325-337. Latour, Bruno (1990), 'Drawing things together' in Michael Lynch and Steve Woolgar (eds.), Representation in Scientific Practice. Cambridge MA: MIT Press, 19-68.

Latour, Bruno (2002), 'What is iconoclash? Or is there a world beyond the image wars?' in Bruno Latour and Peter Weibel (eds.), Iconoclash: Beyond the Image Wars in Science, Religion and Art (Cambridge MA: MIT Press), 16-38.

Lösch, Andreas (2006), 'Anticipating the Futures of Nanotechnology: Visionary Images as Means of Communication'. Technology Analysis \& Strategic Management (Special Issue on the Sociology of Expectations in Science and Technology) 18 (3-4): 393-409.

Lynch, Michael (1985), 'Discipline and the Material Form of Images: An Analysis of Scientific Visibility', Social Studies of Science 15: 37-66.

Lynch, Michael (1990), 'The externalized retina: selection and mathematization in the visual documentation of objects in the life sciences' in Michael Lynch and Steve Woolgar (eds.), Representation in Scientific Practice. Cambridge MA: MIT Press, 153-186.

Lynch, Michael and Steve Woolgar (eds.) (1990a), Representation in Scientific Practice. Cambridge MA: MIT Press.

Lynch, Michael and Steve Woolgar (1990b), 'Introduction: Sociological orientations to representational practice in science' in Michael Lynch and Steve Woolgar (eds.), Representation in Scientific Practice. Cambridge MA: MIT Press, 1-18.

Milburn, Colin (2008), Nanovision: Engineering the Future. Durham \& London: Duke University Press. 
Mody, Cyrus C.M. (2004), 'How probe microscopists became nanotechnologists' in David Baird, Alfred Nordmann and Joachim Schummer (eds.), Discovering the Nanoscale, Amsterdam: IOS Press, 119-133.

Myers, Greg (1990), 'Every picture tells a story: Illustrations in E.O. Wilson's Sociobiology' in Michael Lynch and Steve Woolgar (eds.), Representation in Scientific Practice. Cambridge MA: MIT Press, 231-265.

Nerlich, Brigitte (2008), 'Powered by Imagination: Nanobots at the Science Photo Library', Science as Culture 17 (3): 269-292. Rheinberger, Hans-Jörg (2006), Epistemologie des Konkreten. Frankfurt/Main: Suhrkamp. 


\section{LONDON SCHOOL OF ECONOMICS \\ DEPARTMENT OF ECONOMIC HISTORY}

WORKING PAPERS IN: THE NATURE OF EVIDENCE: HOW WELL DO "FACTS" TRAVEL?

For further copies of this, and to see other titles in the department's group of working paper series, visit our website at: http://www.Ise.ac.uk/collections/economichistory/

2005

01/05: $\quad$ Transferring Technical Knowledge and innovating in Europe, c.1200-c.1800 Stephan R. Epstein

02/05: A Dreadful Heritage: Interpreting Epidemic Disease at Eyam, 1666-2000 Patrick Wallis

03/05: Experimental Farming and Ricardo's Political Arithmetic of Distribution Mary S. Morgan

04/05: $\quad$ Moral Facts and Scientific Fiction: $19^{\text {th }}$ Century Theological Reactions to Darwinism in Germany Bernhard Kleeberg

05/05: Interdisciplinarity "In the Making": Modelling Infectious Diseases Erika Mattila

06/05: $\quad$ Market Disciplines in Victorian Britain Paul Johnson

2006

07/06: Wormy Logic: Model Organisms as Case-based Reasoning Rachel A. Ankeny

08/06: How The Mind Worked: Some Obstacles And Developments In The Popularisation of Psychology Jon Adams 
09/06: $\quad$ Mapping Poverty in Agar Town: Economic Conditions Prior to the Development of St. Pancras Station in 1866 Steven P. Swenson

10/06: "A Thing Ridiculous"? Chemical Medicines and the Prolongation of Human Life in Seventeenth-Century England

David Boyd Haycock

11/06: Institutional Facts and Standardisation: The Case of Measurements in the London Coal Trade.

Aashish Velkar

12/06: Confronting the Stigma of Perfection: Genetic Demography, Diversity and the Quest for a Democratic Eugenics in the Post-war United States Edmund Ramsden

13/06: Measuring Instruments in Economics and the Velocity of Money

Mary S. Morgan

14/06: $\quad$ The Roofs of Wren and Jones: A Seventeenth-Century Migration of Technical Knowledge from Italy to England Simona Valeriani

15/06: Rodney Hilton, Marxism, and the Transition from Feudalism to Capitalism Stephan R. Epstein

2007

16/07: Battle in the Planning Office: Biased Experts versus Normative Statisticians Marcel Boumans

17/07: Trading Facts: Arrow's Fundamental Paradox and the Emergence of Global News Networks, 1750-1900 Gerben Bakker

18/07: Accurate Measurements and Design Standards: Consistency of Design and the Travel of 'Facts' Between Heterogenous Groups Aashish Velkar 
19/07: When Rabbits became Human (and Humans, Rabbits): Stability, Order, and History in the Study of Populations Paul Erickson and Gregg Mitman

20/07: $\quad$ Contesting Democracy: Science Popularisation and Public Choice Jon Adams

21/07 Carlyle and the French Enlightenment: Transitional Readings of Voltaire and Diderot T.J. Hochstrasser

22/02 Apprenticeship and Training in Premodern England Patrick Wallis

2008

23/08 Escaping the Laboratory: The Rodent Experimentsof John B. Calhoun and Their Cultural Influence Edmund Ramsden and Jon Adams

24/08 Travelling in the Social Science Community: Assessing the Impact of the Indian Green Revolution Across Disciplines Peter Howlett

25/08 Circulating Evidence Across Research Contexts: The Locality of Data and Claims in Model Organism Research Sabina Leonelli

26/08 The Lives of 'Facts': Understanding Disease Transmission Through the Case of Haemophilis Influenzae type b Bacteria Erika Mattila

27/08 Regulating Data Travel in the Life Sciences: The Impact of Commodification Sabina Leonelli

28/08 A Journey Through Times and Cultures? Ancient Greek Forms in American Nineteenth Century Architecture: An Archaeological View Lambert Schneider 
29/08 Behind the Facade: Elias Holl and the Italian Influence on Building Techniques in Augsberg

Simona Valeriani

30/08 What Happens to Facts After Their Construction?

Characteristics and Functional Roles of Facts in the

Dissemination of Knowledge Across Modelling

Communities

Erika Mansnerus (formerly Mattila)

31/08 'Voice' and the Facts and Observation of Experience Mary S. Morgan

32/08 Dilemmas in the Constitution of and Exportation of Ethological Facts

Richard Burkhardt

33/08 Travelling with the GDP Through Early Development Economic History

Daniel Speich

34/08 'On A Mission' with Mutable Mobiles

Mary S. Morgan

35/08 Agri-Technologies and Travelling Facts: Case Study of Extension Education in Tamil Nadu, India

Peter Howlett and Aashish Velkar

2009

36/09 Indexing Nature: Carl Linnaeus (1707-1778) and his FactGathering Strategies

Staffan Muller-Wille and Sara Scharf

37/09 Acting with 'Facts' in Order to Re-model Vaccination Policies: The Case of MMR Vaccine in the UK, 1988 Erika Mansnerus

2010

38/10 Designed for Travel: Communicating Facts Through Images

Martina Merz 